\title{
Review
}

\section{PI3K isoform-selective inhibitors: next-generation targeted cancer therapies}

\author{
Xiang WANG, Jian DING*, Ling-hua MENG*
}

Division of Anti-Tumor Pharmacology, State Key Laboratory of Drug Research, Shanghai Institute of Materia Medica, Chinese Academy of Sciences, Shanghai 201203, China

\begin{abstract}
The pivotal roles of phosphatidylinositol 3-kinases (PI3Ks) in human cancers have inspired active development of small molecules to inhibit these lipid kinases. However, the first-generation pan-PI3K and dual-PI3K/mTOR inhibitors have encountered problems in clinical trials, with limited efficacies as a monotherapeutic agent as well as a relatively high rate of side effects. It is increasingly recognized that different PI3K isoforms play non-redundant roles in particular tumor types, which has prompted the development of isoform-selective inhibitors for pre-selected patients with the aim for improving efficacy while decreasing undesirable side effects. The success of PI3K isoform-selective inhibitors is represented by CAL101 (Idelalisib), a first-in-class PI3Kס-selective small-molecule inhibitor that has been approved by the FDA for the treatment of chronic lymphocytic leukemia, indolent B-cell non-Hodgkin's lymphoma and relapsed small lymphocytic lymphoma. Inhibitors targeting other PI3K isoforms are also being extensively developed. This review focuses on the recent progress in development of PI3K isoform-selective inhibitors for cancer therapy. A deeper understanding of the action modes of novel PI3K isoform-selective inhibitors will provide valuable information to further validate the concept of targeting specific PI3K isoforms, while the identification of biomarkers to stratify patients who are likely to benefit from the therapy will be essential for the success of these agents.
\end{abstract}

Keywords: PI3K isoforms; isoform-selective inhibitor; CAL101; precise cancer therapy; biomarker

Acta Pharmacologica Sinica (2015) 36: 1170-1176; doi: 10.1038/aps.2015.71; published online 14 Sep 2015

\section{Introduction}

Phosphatidylinositol 3-kinases (PI3Ks) belong to a family of lipid kinases that are named after their ability to phosphorylate the 3'-hydroxyl group of phosphatidylinositol (PtdIns) lipids to produce the second messengers PtdIns(3)P, PtdIns $(3,4)$ P2 and/or PtdIns(3,4,5)P3 (PIP3) on the cell membrane. PI3Ks retain in the cytosol with low activity, but their activity can be dramatically elevated by inputs from the cell membrane or the cytosol, which orchestrates multiple signaling pathways and regulates various cellular processes. The PI3K-AktmTOR pathway is one of the key axes mediated by PI3Ks and it has been well elucidated (Figure 1). Cellular membraneassociated PIP3 recruits Akt (also known as protein kinase $\mathrm{B}, \mathrm{PKB})$, a serine/threonine protein kinase that contains a pleckstrin homology $(\mathrm{PH})$ domain. The binding of Akt to PIP3 leads to its conformational change, and Akt is activated following phosphorylation by 3 '-phosphoinositide dependent

\footnotetext{
* To whom correspondence should be addressed.

E-mail Ihmeng@simm.ac.cn (Ling-hua MENG) jding@simm.ac.cn (Jian DING)

Received 2015-05-30 Accepted 2015-07-12
}

protein kinase-1 (PDK1) at Thr308 and by mammalian target of rapamycin complex 2 (mTORC2) at Ser473. Akt subsequently regulates cell cycle progression and glucose metabolism by further phosphorylating its numerous substrates, such as FOXOs, GSK3 and AS160. Akt also activates mammalian target of rapamycin complex 1 (mTORC1) in multiple ways and regulates protein synthesis. Phosphatase and tensin homolog (PTEN), a lipid phosphatase, stops this process by dephosphorylating the $3^{\prime}$ phosphate group of PIP3. The PI3K pathway is frequently deregulated in human tumors due to genetic and epigenetic aberrations of key components in the pathway or upstream regulators. Although great progress has been made to develop therapeutics targeting the PI3KAkt-mTOR pathway in past decades, it has been increasingly recognized that distinct isoforms in the PI3K family play nonredundant roles in physiological situations and tumors, which has prompted the development of isoform-selective inhibitors for pre-selected patients, aimed at improving efficacy while minimizing undesirable side effects.

\section{Classification of PI3Ks}

The PI3K family is divided into three classes (I, II, III) based 


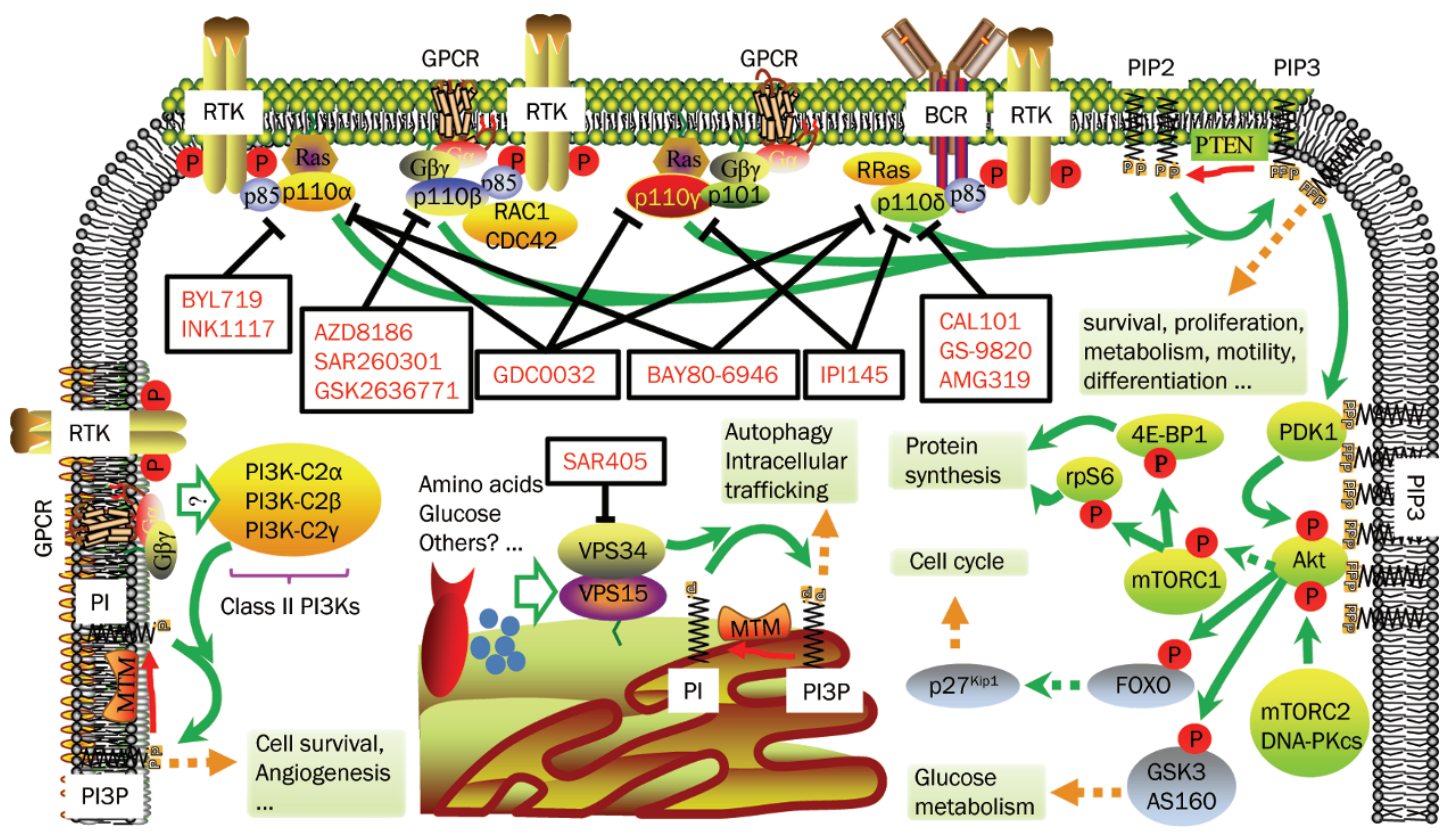

Figure 1. Divergent regulation of PI3K isoforms and isoform-selective inhibitors. PI3K $\alpha$ is activated by RTKs and Ras; PI3K $\beta$ is the downstream kinase of RTKs, GPCRs and RAC1/CDC42; PI3Ky mediates signals from GPCRs and Ras, while PI3Kס is under the control of RTKs and antigen receptors. Class I PI3Ks produce the second messenger PIP3, which promotes cell survival, proliferation, metabolism, motility and differentiation. One of the key mediators is Akt, which is phosphorylated and activated by PDK1 and mTORC2 or DNA-PKcs in the presence of PIP3. Akt further phosphorylates diversified substrates and regulates protein synthesis, cell cycle and glucose metabolism. PTEN acts as a negative regulator and removes the $3^{\prime}$ phosphate from PIP3. The potential inputs of class II PI3Ks include GPCRs and RTKs, and their product Ptdlns(3)P (PI3P) has a role in cell survival and angiogenesis. Amino acids, glucose and certain GPCRs are reported as stimuli of Vps34, which is able to produce PI3P to regulate autophagy and intracellular trafficking. The MTM phosphatase family removes 3' phosphate from PI3P and terminates its signaling. Representative inhibitors of each isoforms are highlighted in red and are listed in Table 1.

on their coding genes, distinct structures and substrate preference. There are four members in class I, with the subdivisions of PI3Ka, PI3K $\beta$ and PI3K $\delta$ into class IA and PI3KY into class IB, according to their activating models ${ }^{[1]}$. Class IA PI3Ks are heterodimers of one p110 catalytic subunit encoded by PIK3CA (p110a), PIK3CB (p110 $\beta$ ) or PIK3CD (p1108) and one p85 regulatory subunit encoded by PIK3R1 (p85a), PIK3R2 (p85 $\beta$ ) or PIK3R3 (p85y). PI3K $\gamma$ is also a heterodimer enzyme containing the catalytic subunit $\mathrm{p} 110 \mathrm{\gamma}$ encoded by PIK3CG and one regulatory subunit p101 encoded by PIK3R5 or 884 (also known as p87 $7^{\text {PIKAP }}$ ) encoded by PIK3R6. PI3Ka and PI3K $\beta$ are ubiquitously expressed in all cells and tissues, whereas PI3K $\gamma$ and PI3K $\delta$ are mainly enriched in leukocytes. In quiescent settings, class I PI3Ks possess little kinase activity due to the self-inhibition of p85 regulatory subunits and/or localization in the cytosol, where no substrates are available. Upon activation via binding of receptor tyrosine kinase (RTK), G protein-coupled receptor (GPCR), Ras or other adaptor proteins, class I PI3Ks are recruited to the cell membrane proximity, where they switch to an active conformation and utilize PtdIns $(4,5)$ P2 to generate PIP3.

Class II PI3Ks are monomeric enzymes containing three isoforms, PI3K-C2 $\alpha$, PI3K-C2 $\beta$ and PI3K-C $2 \gamma$, which are encoded by PIK3C2A, PIK3C3B and PIK3C2G, respectively. These isoforms are able to preferentially convert PtdIns into PtdIns(3)P in cells. PI3K-C2 $\alpha$ and PI3K-C2 $\beta$ are widely distributed in tissues but $\mathrm{PI} 3 \mathrm{~K}-\mathrm{C} 2 \gamma$ is mainly restricted to the liver, prostate and breast tissues. Although class II PI3Ks appear to be modestly activated by receptor tyrosine kinases and GPCR, their regulations and physiological functions are relatively unclear.

Class III PI3K has one member named after its catalytic subunit, Vps34, which is encoded by PIK3C3. Vps34 couples to its regulatory subunit Vps15 (encoded by PIK3R4) to form a heterodimer and is membrane-tethered due to the $\mathrm{N}$-terminal myristoylation sequence in Vps15. Vps34 is ubiquitously expressed, and its product PtdIns(3)P is also a lipid messenger that regulates intracellular trafficking and autophagy. It has been reported that Vps34 activity is modulated by nutrients, such as amino acids and glucose, and by GPCRs. The myotubularin family phosphatase MTM1 and MTMR1 serve as negative regulators against class II and III PI3Ks by dephosphorylating the 3' phosphate group in PtdIns(3)P.

Among the eight PI3Ks, class I PI3Ks have been the most studied because of their fundamental physiological and pathological roles. Aberrant PI3K activities are frequently observed in many types of cancers through different mechanisms including (but not limited to) hyperactivated RTKs, mutant Ras, functional loss of PTEN and activating mutations and/or overexpression of PI3K isoforms. PIK3CA is mutated across different tumors with a frequency of approximately one-third in endometrial, breast, ovarian and colorectal cancer specimens. The majority of mutations are clustered within exons 10 
and 21 (the helical domain and kinase domain, respectively), with $80 \%$ of the identified mutations found within three 'hotspots', E542, E545, and H1047, which dramatically increase PI3K kinase activity and were shown to be oncogenic ${ }^{[2]}$. Activating mutations are detected in PIK3CB and PIK3CD with rare frequency. Somatic alternations in the regulatory subunits PIK3R1 and PIK3R2 with reduced inhibitory capability against p110 have been identified by large-scale cancer genomic sequencing ${ }^{[3]}$. Amplification or over expression of PIK3CA, PIK3CB, PIK3CD or PIK3CG are also commonly detected in tumor specimen and cultured tumor cells. Functional loss or decrease of PTEN also leads to hyper-activated PI3K signaling in certain tumors.

PI3K have been validated as promising anticancer targets, and tremendous efforts have been put to develop small- molecule inhibitors for cancer therapy. However, the objective response of the first-generation pan-PI3K inhibitors or dualPI3K/mTOR inhibitors as monotherapeutic agents has been modest ${ }^{[4]}$. With the new knowledge regarding the divergent roles of PI3K isoforms in different types of cancer, isoformselective PI3K inhibitors have attracted increasing interest for precise cancer therapy, and over a dozen inhibitors are undergoing clinical evaluation (Table 1). Among them, CAL101 has been approved by the U.S. Food and Drug Administration (FDA) for patients with relapsed chronic lymphocytic leukemia (CLL) and indolent lymphoma.

\section{PI3Ko-selective inhibitors}

The importance and high frequency of PIK3CA mutation in solid tumors has attracted attention towards the development

Table 1. Isoform-selective PI3K inhibitors.

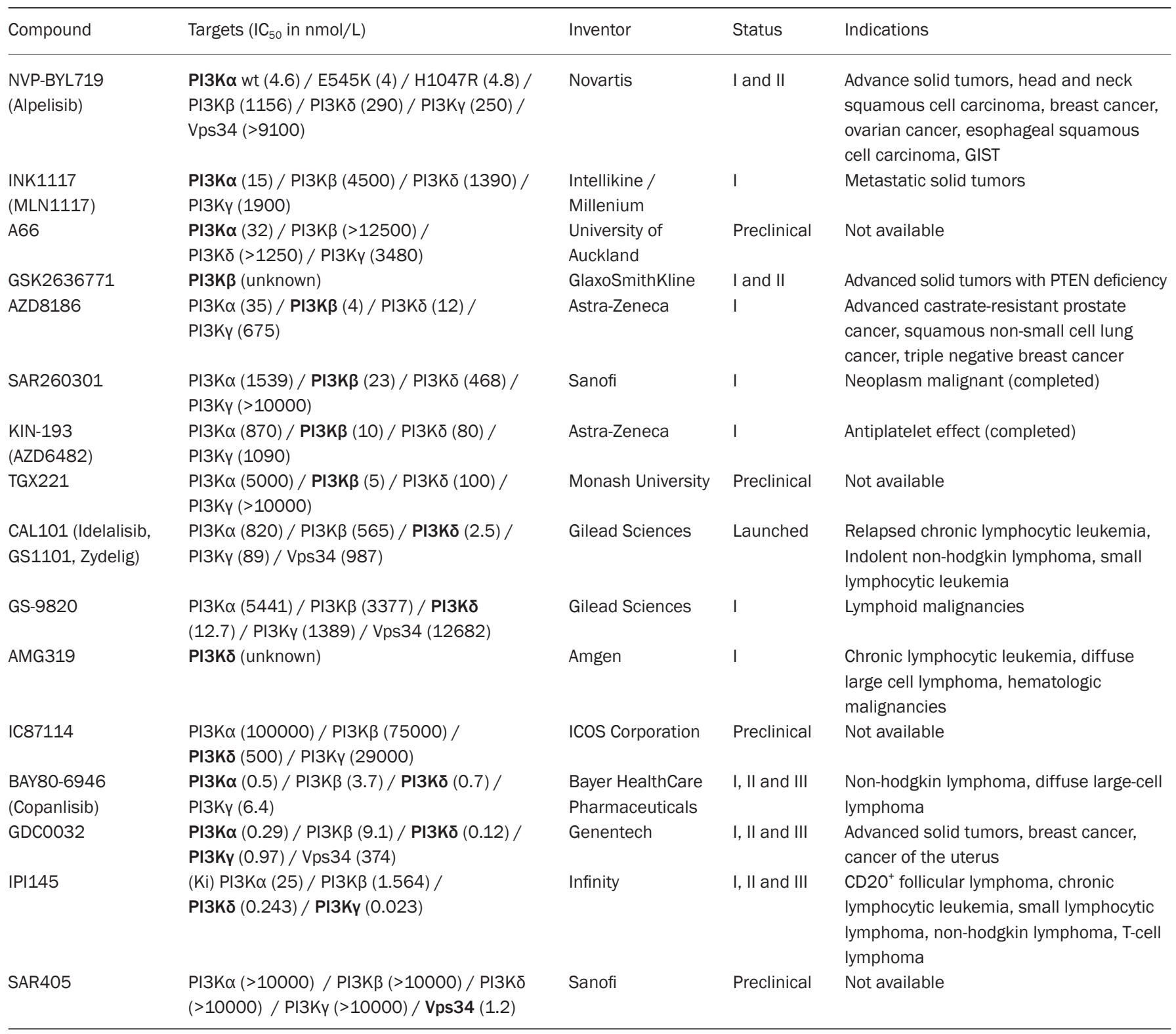


of PI3Ka-selective inhibitors. A66 derived from a pan-PI3K inhibitor PIK-93 has emerged as one of the most specific PI3Ka inhibitors with a similar potency against wild type PI3Ka, E545K and H1047R mutants but not targeting other PI3Ks and protein kinases ${ }^{[5]}$. A66 potently inhibits the proliferation of PI3K-mutant cells in vitro and in vivo, but its short half-life impeded its further clinical development. Further structural modifications based on A66 resulted in NVP-BYL719, which possesses optimal PI3Ka selectivity and potency with better pharmacokinetic properties. NVP-BYL719 is currently being tested for the treatment of PIK3CA-mutated advanced solid tumors as a monotherapy or head and neck squamous cell carcinoma, advanced breast cancer, etc. as part of a combinatorial approach (https://www.clinicaltrials.gov/). The results from the first-in-human study demonstrated that NVP-BYL719 displayed dose-proportional and predictable pharmacokinetics. The safety profile is favorable, with manageable on-target toxicities. At doses over $270 \mathrm{mg} / \mathrm{d}$, tumor regression and prolonged disease control were observed in heavily pretreated patients with various types of tumor carrying a PIK3CA mutation ${ }^{[6]}$.

INK1117 is another reported PI3Ka inhibitor in phase I clinical trials for treatment of solid tumors. We also have discovered a potent PI3Ka inhibitor in preparation for its application in clinical trials (unpublished data). PI3Ka-mutant specific inhibitors have also been identified (personal communication with Dr Ming-wei WANG in the Chinese National Compound Library).

The PI3Ka inhibitor treatment results in $\mathrm{G}_{1}$ phase arrest without killing cells in vitro ${ }^{[7]}$, which is consistent with lack of tumor regression in clinical settings. Sporadic studies have indicated the induction of apoptosis by NVP-BYL719, but this effect appears to be dependent on cell types ${ }^{[8]}$. Recent preclinical studies have found that the growth of HER2- or KRAS-driven solid tumors highly relies on PI3Ka, and the inhibition of this isoform is sufficient to halt tumor growth to an extent similar to that of blocking all class I isoforms ${ }^{[9,}$ ${ }^{10]}$, underscoring PI3Ka as a promising target in these types of tumors. Moreover, PI3Ka is important for angiogenesis in solid tumors, which may suffer from a deficient blood supply upon inhibition of this isoform. The function of PI3Ka in cell metabolism regulation has been observed to promote cancer cell survival. In addition, decreased glucose consumption is considered a positive sign in predicting the antitumor effect of NVP-BYL719. PI3Ka has recently been found to play roles in immune modulation. The inhibition of PI3Ka may either suppress or enhance immune responses in a diverse subset of innate and adaptive immune cells ${ }^{[11,12]}$. It is important to better understand the mechanistic action of PI3Ka inhibitors on the immune system to harness immune effects to create a more favorable immune environment for cancer therapy. Consistent with its high selectivity against PI3Ka, a study analyzing the effects of NVP-BYL719 on a compilation of cell lines as well as on a panel of PDX models reveals that a PIK3CA mutation was the foremost positive predictor of drug sensitivity. PIK3CA amplification is an additional positive predicator, while PTEN mutation is a negative predicator of drug sensitivity ${ }^{[13]}$. In line with this finding, patients harboring the PIK3CA H1047R mutation were found to be the most responsive cohort in early clinical trials, while PTEN loss was associated with resistance to NVP-BYL719 ${ }^{[14]}$. A recent study found that the initial efficacy of PI3Ka inhibition was mitigated by the rapid re-accumulation of PIP3 produced by the PI3K $\beta$ isoform, which attenuated the efficacy of NVP-BYL719 in breast cancer cells. It is not known whether this phenomenon is shared in other cancer types. Nevertheless, the mechanism of action of PI3Ka inhibitors in different cancer types and genetic contexts should be further elucidated, which will facilitate the identification of biomarkers predicting efficacy and also combination regimens.

\section{PI3Kס-selective inhibitors}

PI3K is the primary PI3K isoform in leukocytes that mediates signals from RTKs and tyrosine-based activation motif (ITAM)-containing proteins because of its high enrichment in these cells ${ }^{[15]}$. Pharmacological inactivation of PI3K $\delta$ reveals its importance for the function of $\mathrm{T}$ cells, B cells, mast cells and neutrophils. Hyper-activated PI3K signaling is a common event in leukemia specimens and cultured cells. Hence, targeting PI3K $\delta$ may be beneficial both for auto-immune diseases and cancer.

IC87114 is the first reported isoform-selective PI3K inhibitor against PI3K $\delta$ though random screening of a diversified chemical library. IC87114 selectively inhibits PI3K $\delta$ with an $\mathrm{IC}_{50}$ of $0.5 \mu \mathrm{mol} / \mathrm{L}$, which is 58 -fold lower than that against PI3KY and over 100-fold lower than those against PI3Ka, PI3K $\beta$ and a panel of protein kinases. Further modifications based on IC87114 resulted in CAL101 with improved potency against PI3K $\delta\left(\mathrm{IC}_{50}=15 \mathrm{nmol} / \mathrm{L}\right)$ and selectivity over other class I isoforms (453-, 210-, and 110-fold selective against PI3Ka, PI3K $\beta$, and PI3KY isoforms, respectively) and other 351 kinases $^{[16]}$. Co-crystallization of p110ठ and IC87114/CAL101 revealed that binding of IC87114/CAL101 with p1108 forces the latter to open a hydrophobic pocket with high affinity with fluoroquinazolinone group of the inhibitors ${ }^{[17,18]}$. However, PI3K $\delta$ inhibitors with different binding models have also been reported, such as X-370 ${ }^{[19]}$. The detailed SARs of PI3K $\delta$ inhibitors have been described previously ${ }^{[20]}$.

Because PI3Kס is a key component in the signaling in B cell malignancies and is located downstream of various $B$ cell receptors, including the $\mathrm{B}$ cell antigen receptor (BCR), cytokine/chemokine receptors, and adhesion molecules, CAL101 was tested in a subset of patients with indolent B cell malignancies and dramatic and durable responses were observed in phase I clinical trials, leading to an expanded phase II, uncontrolled trial of CAL101 monotherapy in indolent nonHodgkin's lymphoma patients. The overall response rate and the median progression-free survival was $57 \%$ and 11 months, respectively, illustrating the similar or superior efficacy of CAL101 to those of other active medications. In a randomized, double-blind phase III clinical trial in relapsed chronic lymphocytic leukemia, patients received rituximab with either 
CAL101 or placebo. The overall response rate was $81 \%$ and $13 \%$ in the CAL101 combination group and the placebo control group, respectively. The median progression-free survival was 5.5 months with rituximab only, but was not reached with the CAL101 combination. CAL101 plus rituximab was effective in patients with CLL who not only were heavily pretreated but also had high-risk genetic profiles, including p53 loss. The grade 3 or higher adverse events of CAL101 treatment were neutropenia (27\% patients), aminotransferase level elevation (13\% patients), diarrhea (13\% patients) and pneumonia (7\% patients), but the events were manageable ${ }^{[21,22]}$. GS-9820 and AMG319 are other two PI3Kס-selective inhibitors currently undergoing phase I trials for lymphoid malignancies $^{[23]}$.

Although oncogenic mutations or overexpression of PIK3CD has not been found in CLL tumor cells and primary patient samples, increased PI3K activity has been observed, which is highly dependent on the PI3K $\delta$ isoform. PI3K $\delta$ inhibitors attenuate survival signals by blocking constitutive PI3K signaling including phosphorylation of AKT and ERK1/2. It has been reported that CAL101 down-regulates the secretion of chemokines and inhibits CLL cell chemotaxis towards CXCL12 and CXCL13. In addition, CAL101 abrogates protection from spontaneous apoptosis induced by B cell-activating factors CD40L, TNF-a, and fibronectin. In line with these findings, CAL101 treatment significantly reduced levels of circulating CCL3, CCL4, CXCL13 and lymphocytes in clinical settings. High concentrations and long-term treatment are required for PI3K $\delta$ inhibitors to induce apoptosis in vitro, suggesting that the induction of apoptosis is unlikely the direct mechanism to exert anti-cancer activity. Thus, PI3K $\delta$ inhibitors may display dual mechanisms to directly decrease tumor cell survival and to reduce survival signals in the microenvironment ${ }^{[24-26]}$.

The life cycle of CLL cells involves homing and egression in patients. The migration of leukemic cells from the peripheral blood to the lymph nodes, spleen, and bone marrow, where they are become activated by micro-environmental stimuli, leads to survival and proliferation. Inhibition of PI3Kठ may interfere with this cycling at various levels, resulting in the mobilization of tissue-resident CLL cells into the blood and blocking re-entry to the stromal. Sustained interruption of this recirculation cycle may disrupt survival signals and sensitize the cells to combination treatments. It is likely that the dynamic life cycle of CLL could explain why this disease, among other the different $B$ cell malignancies, is the most responsive to $\mathrm{B}$ cell signaling blockers. Residual PI3K activity present in immune cells upon PI3Kס inhibition by CAL101, but not other broad-spectrum PI3K inhibitors, may explain its lack of overt immune suppression upon long-term administration $^{[27,28]}$. In addition, evidence from another PI3K $\delta$ inhibitor, PI-3065, suggests that patients with solid tumors may also benefit from PI3K $\delta$ inhibition, as inactivation of PI3K $\delta$ hinders tumor growth by circumventing regulatory $\mathrm{T}$ cell-mediated immune tolerance ${ }^{[29]}$. Our group found that a novel PI3K inhibitor, X-370, preferentially inhibited survival of primary $\mathrm{B}$ cell acute lymphoblast leukemia (B-ALL) cells harboring
PI3K-dependent Erk1/2 phosphorylation via a PI3K-PDK1MEK1/2-Erk1/2 signaling cascade. The existence of this signaling pathway may possibly be used to identify responsive B-ALL patients.

The findings from the laboratory bench translated well into bedside treatment and vice versa, leading to the final approval of CAL101. However, the exact mechanisms of PI3Kס inhibitors for the treatment of B-cell type leukemia and possibly solid tumors have not been fully elucidated. A deeper understanding of the modes of action of PI3K $\delta$ inhibitors will provide valuable clues to further validate the PI3K $\delta$-targeting approach and to identify biomarkers capable of stratifying patients who are likely to benefit from the therapy. Moreover, the identification of predictive biomarkers capable of monitoring the efficacy of PI3Kס inhibitors in clinical is urgently needed for different types of tumors.

\section{Other PI3K isoform-selective inhibitors}

PI3K $\beta$ is another ubiquitously expressed class I PI3K in addition to PI3Ka. Overexpression of $\mathrm{p} 110 \beta$ is sufficient to transform chicken embryo fibroblasts, and the knockout of PIKЗСB circumvents tumor formation in PTEN-null prostate cancer mouse models. PTEN loss or mutation is detected in a considerable fraction $(20 \%-75 \%)$ of tumors, including gliomas, breast, colon, lung, endometrial and prostate cancers. Hence, PI3K $\beta$ has been recognized as a therapeutic target in this subset of solid tumors ${ }^{[30]}$.

TGX221, a PI3K $\beta$-selective inhibitor derived from LY294002, has been a desirable template for further optimization. The TGX221 analogues KIN-193 (AZD6482) and SAR260301 have been developed for the treatment of thrombosis and solid tumors, respectively ${ }^{[31]}$. PI3K $\beta$ inhibitors have been shown to selectively inhibit the growth of tumor cells and xenografts deficient in PTEN, which prompted a new clinical trial to investigate the efficacy of the PI3K $\beta$-selective inhibitor GSK2636771 in patients with PTEN-null advanced solid tumors. However, targeting PI3K $\beta$ in PTEN-deficient tumors may be compromised by tumor heterogeneity, coexisting genetic alterations and micro-environmental factors. For example, the presence of oncogenic RTKs, RAS or PIK3CA mutations may shift dependency partially or totally to PI3Ka in PTEN-deficient tumors. Moreover, the prolonged treatment of PTEN-deficient tumor cells with a PI3K $\beta$ inhibitor may also shift isoform dependency from PI3K $\beta$ to PI3Ka ${ }^{[32]}$. Interestingly, the treatment of glioblastoma cells deficient in PTEN with CAL101, but not PI3K $\beta$ inhibitors, resulted in decreased cell migration ${ }^{[33]}$. A recent study demonstrated PTEN-mutated endometrial cancer cells are resistant to GSK2636771 and KIN193, while co-treatment of the PI3Ka-selective inhibitor A66 resulted in a decrease in cell viability ${ }^{[34]}$. These results suggest that pan-PI3K inhibitors may be more effective in PTENdeficient tumors than isoform-selective inhibitors.

The loss of PIK3CG dampens thymocyte development and $\mathrm{T}$ cell viability in mouse models, indicating its role in inflammation. Overexpression of $\mathrm{p} 110 \mathrm{y}$ initiates oncogenic transformation. With the validation of $\mathrm{PI} \mathrm{K}_{\gamma}$ as a promising drug 
target for the treatment of inflammatory disease and, possibly, leukemia, a number of PI3K $\gamma$-selective inhibitors have been discovered, but none has advanced to clinical trials. Although a direct link between tumorigenesis and class II/III PI3Ks has not been reported, a few studies have discovered amplification or mutation of PIK3C2B in cancers. Vps34 has been recently reported to be required for the cellular transformation of oncoprotein $S R C^{[35]}$ in addition to its important roles in autophagy. SAR405 has been reported recently to inhibit Vps34 and exert synergistic antiproliferative activity in combination with mTOR inhibitor everolimus in renal cancer cells ${ }^{[36]}$. Moreover, dual- or triple-isoform-selective inhibitors have also been discovered for cancer treatment such as BAY80-6946 (PI3Ka/

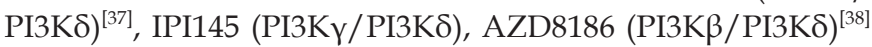
and GDC0032 (PI3Ka/PI3K $\gamma / \mathrm{PI} 3 \mathrm{~K} \delta)^{[39]}$. The divergent selectivity profiles fit well with the complexity of human tumors, and this advantage should be taken to devise an optimal therapeutic strategy with precise therapy.

\section{Conclusions and perspectives}

Accumulated knowledge from years of basic research allows us to appreciate the complex signaling network transduced by PI3Ks. The distinct and convergent roles of individual PI3K isoform are being uncovered. Isoform-selective inhibitors are emerging as a next-generation of PI3K inhibitors with improved, precise targeting and reduced toxicity, which is exemplified by the success of CAL101 in CLL therapy. However, targeting PI3Ks alone is unlikely to be a curative therapy for diverse cancers, and further efforts in biomarker discovery, rational combinations and basic research are warranted.

Though the divergent roles of each isoforms in different signaling contexts, various tissues and types of diseases have been extensively studied, continuous efforts need to be made to explore the precise functions among PI3K isoforms in order to implement precise targeting. The mechanism of action of isoform-selective inhibitors needs to be extensively and systematically studied, which will allow us not only to monitor efficacy and side effect, but also to develop personalized therapeutic strategy. Of particular note, cancer is also a disease related to immune adaptation and efforts should be made to understand how specific PI3K inhibitors re-modulate immune environment in tumor therapy. The close relationship between PI3K and cell metabolism should also be reconsidered in the big picture of cancer therapies.

In consistency with the high specificity of PI3K isoformselective inhibitors, they are expected to target a subset of tumors. A number of biomarkers which are capable of predicting the efficacy of PI3K isoform-selective inhibitors have been identified and some of them have been validated in clinical settings. However, these biomarkers need to be tested in large scale clinical trials. As different PI3K isoform-selective inhibitors display different spectra for tumor therapy, different biomarkers are warranted to be identified for the treatment of different types of tumors. With recent advances in the next generation sequencing and clinical trials under the concept of precision medicine, predictive biomarkers for the efficacy of each type of inhibitors in a given tumor are expected to be identified and validated. It remains unclear whether preclinical observations of improved responses to PI3K isoformselective inhibitors in solid tumors with PIK3CA and PTEN alterations will be born out in clinical trials.

Preliminary clinical data indicates that PI3Ka inhibitors achieve modest responses as monotherapy. Researchers need to endeavor in figuring out mechanisms underlying drug resistance and escape of PI3K dependency in PI3K aberrantly activated tumors, thus facilitate rational combination therapies to improve the efficacy. Good tolerability of PI3K isoformselective inhibitors in patients allows broad combinations with conventional radio- and chemo-therapy and other molecularly targeted therapy in order to obtain durable response. However, successful combination regimens are based on understanding the molecular alterations in given tumors.

In conclusion, next-generation PI3K isoform-selective inhibitors provide both opportunities and challenges for cancer therapy within the realm of precision medical treatments. With continuous drive and commitment in this important field, increasing numbers of patients are expected to benefit from therapy based on PI3K isoform-selective inhibitors in the near future.

\section{Acknowledgements}

This research was supported by National Science \& Technology Major Project "Key New Drug Creation and Manufacturing Program" (2012ZX09301-001), National Natural Science Foundation of China (81321092, 81373445, and 81402972) and Science and Technology Commission of Shanghai Municipality (14431905200).

\section{References}

1 Vanhaesebroeck B, Guillermet-Guibert J, Graupera M, Bilanges B. The emerging mechanisms of isoform-specific $\mathrm{PI} 3 \mathrm{~K}$ signalling. Nat Rev Mol Cell Biol 2010; 11: 329-41.

2 Rudd ML, Price JC, Fogoros S, Godwin AK, Sgroi DC, Merino MJ, et al. A unique spectrum of somatic PIK3CA (p110alpha) mutations within primary endometrial carcinomas. Clin Cancer Res 2011; 17: 133140.

3 Cheung LW, Yu S, Zhang D, Li J, Ng PK, Panupinthu N, et al. Naturally occurring neomorphic PIK3R1 mutations activate the MAPK pathway, dictating therapeutic response to MAPK pathway inhibitors. Cancer Cell 2014; 26: 479-94.

4 Rodon J, Dienstmann R, Serra V, Tabernero J. Development of PI3K inhibitors: lessons learned from early clinical trials. Nat Rev Clin Oncol 2013; 10: 143-53.

5 Jamieson S, Flanagan JU, Kolekar S, Buchanan C, Kendall JD, Lee WJ, et al. A drug targeting only p110alpha can block phosphoinositide 3-kinase signalling and tumour growth in certain cell types. Biochem J 2011; 438: 53-62.

6 Ana M. Gonzalez-Angulo DJ, Guillem A, Jan HM S, Burris HA, Berlin $J$, et al. Safety, pharmacokinetics, and preliminary activity of the $\alpha$-specific PI3K inhibitor BYL719: Results from the first-in-human study. J Clin Oncol 2013; 31: abstr 2531.

7 Liu JL, Gao GR, Zhang X, Cao SF, Guo CL, Wang X, et al. DW09849, a selective phosphatidylinositol 3-Kinase (PI3K) inhibitor, prevents $\mathrm{PI} 3 \mathrm{~K}$ signaling and preferentially inhibits proliferation of cells containing 
the oncogenic mutation p110alpha (H1047R). J Pharmacol Exp Ther 2014; 348: 432-41.

8 Gobin B, Huin MB, Lamoureux F, Ory B, Charrier C, Lanel R, et al. BYL719, a new alpha-specific PI3K inhibitor: single administration and in combination with conventional chemotherapy for the treatment of osteosarcoma. Int J Cancer 2015; 136: 784-96.

9 Castellano E, Sheridan C, Thin MZ, Nye E, Spencer-Dene B, Diefenbacher ME, et al. Requirement for interaction of PI3-kinase p110alpha with RAS in lung tumor maintenance. Cancer Cell 2013; 24: 617-30.

10 Gritsman K, Yuzugullu H, Von T, Yan H, Clayton L, Fritsch C, et al. Hematopoiesis and RAS-driven myeloid leukemia differentially require PI3K isoform p110alpha. J Clin Invest 2014; 124: 1794-809.

11 Yea SS, So L, Mallya S, Lee J, Rajasekaran K, Malarkannan S, et al. Effects of novel isoform-selective phosphoinositide 3-kinase inhibitors on natural killer cell function. PLoS One 2014; 9: e99486.

12 Ogino S, Galon J, Fuchs CS, Dranoff G. Cancer immunology--analysis of host and tumor factors for personalized medicine. Nat Rev Clin Oncol 2011; 8: 711-9.

13 Fritsch C, Huang A, Chatenay-Rivauday C, Schnell C, Reddy A, Liu M, et al. Characterization of the novel and specific PI3Kalpha inhibitor NVP-BYL719 and development of the patient stratification strategy for clinical trials. Mol Cancer Ther 2014; 13: 1117-29.

14 Juric D, Castel P, Griffith M, Griffith OL, Won HH, Ellis H, et al. Convergent loss of PTEN leads to clinical resistance to a $\mathrm{PI}(3)$ Kalpha inhibitor. Nature 2015; 518: 240-4.

15 Wang X, Li JP, Yang Y, Ding J, Meng LH. A pharmacological model reveals biased dependency on PI3K isoforms for tumor cell growth. Acta Pharmacol Sin 2013; 34: 1201-7.

16 Ikeda H, Hideshima T, Fulciniti M, Perrone G, Miura N, Yasui H, et al. $\mathrm{PI} 3 \mathrm{~K} / \mathrm{p} 110$ delta\} is a novel therapeutic target in multiple myeloma. Blood 2010; 116: 1460-8.

17 Somoza JR, Koditek D, Villasenor AG, Novikov N, Wong MH, Liclican A, et al. Structural, biochemical, and biophysical characterization of idelalisib binding to phosphoinositide 3-Kinase delta. J Biol Chem 2015; 290: 8439-46.

18 Berndt A, Miller S, Williams O, Le DD, Houseman BT, Pacold Jl, et al. The p110 delta structure: mechanisms for selectivity and potency of new PI(3)K inhibitors. Nat Chem Biol 2010; 6: 117-24.

19 Wang X, Zhang X, Li BS, Zhai X, Yang Z, Ding LX, et al. Simultaneous targeting of PI3Kdelta and a PI3Kdelta-dependent MEK1/2-Erk1/2 pathway for therapy in pediatric B-cell acute lymphoblastic leukemia. Oncotarget 2014; 5: 10732-44.

20 Wei M, Wang X, Song Z, Jiao M, Ding J, Meng LH, et al. Targeting PI3Kdelta: emerging therapy for chronic lymphocytic leukemia and beyond. Med Res Rev 2015; 35: 720-52.

21 Furman RR, Sharman JP, Coutre SE, Cheson BD, Pagel JM, Hillmen $P$, et al. Idelalisib and rituximab in relapsed chronic lymphocytic leukemia. N Engl J Med 2014; 370: 997-1007.

22 Gopal AK, Kahl BS, de Vos S, Wagner-Johnston ND, Schuster SJ, Jurczak WJ, et al. PI3Kdelta inhibition by idelalisib in patients with relapsed indolent lymphoma. N Engl J Med 2014; 370: 1008-18.

23 Shugg RP, Thomson A, Tanabe N, Kashishian A, Steiner BH, Puri $K D$, et al. Effects of isoform-selective phosphatidylinositol 3-kinase inhibitors on osteoclasts: actions on cytoskeletal organization, survival, and resorption. J Biol Chem 2013; 288: 35346-57.

24 Lannutti BJ, Meadows SA, Herman SE, Kashishian A, Steiner B, Johnson AJ, et al. CAL-101, a p110delta selective phosphatidylinositol3-kinase inhibitor for the treatment of B-cell malignancies, inhibits PI3K signaling and cellular viability. Blood 2011; 117: 591-4.
25 Herman SE, Gordon AL, Wagner AJ, Heerema NA, Zhao W, Flynn JM, et al. Phosphatidylinositol 3-kinase-delta inhibitor CAL-101 shows promising preclinical activity in chronic lymphocytic leukemia by antagonizing intrinsic and extrinsic cellular survival signals. Blood 2010; 116: 2078-88.

26 Hoellenriegel J, Meadows SA, Sivina M, Wierda WG, Kantarjian H, Keating MJ, et al. The phosphoinositide 3'-kinase delta inhibitor, CAL101, inhibits B-cell receptor signaling and chemokine networks in chronic lymphocytic leukemia. Blood 2011; 118: 3603-12.

27 lyengar S, Clear A, Bodor C, Maharaj L, Lee A, Calaminici M, et al. P110alpha-mediated constitutive PI3K signaling limits the efficacy of p110delta-selective inhibition in mantle cell lymphoma, particularly with multiple relapse. Blood 2013; 121: 2274-84.

28 Ramadani F, Bolland DJ, Garcon F, Emery JL, Vanhaesebroeck B, Corcoran $\mathrm{AE}$, et al. The PI3K isoforms p110alpha and p110delta are essential for pre-B cell receptor signaling and B cell development. Sci Signal 2010; 3: ra60.

29 Ali K, Soond DR, Pineiro R, Hagemann T, Pearce W, Lim EL, et al. Inactivation of $\mathrm{PI}(3) \mathrm{K}$ p110delta breaks regulatory T-cell-mediated immune tolerance to cancer. Nature 2014; 510: 407-11.

$30 \mathrm{Ni} J$, Liu Q, Xie S, Carlson C, Von T, Vogel K, et al. Functional characterization of an isoform-selective inhibitor of PI3K-p110beta as a potential anticancer agent. Cancer Discov 2012; 2: 425-33.

31 Certal V, Carry JC, Halley F, Virone-Oddos A, Thompson F, FilocheRomme B, et al. Discovery and optimization of pyrimidone indoline amide PI3Kbeta inhibitors for the treatment of phosphatase and tensin homologue (PTEN)-deficient cancers. J Med Chem 2014; 57 : 903-20.

32 Schwartz S, Wongvipat J, Trigwell CB, Hancox U, Carver BS, RodrikOutmezguine V, et al. Feedback suppression of PI3Kalpha signaling in PTEN-mutated tumors is relieved by selective inhibition of PI3Kbeta. Cancer Cell 2015; 27: 109-22.

33 Luk SK, Piekorz RP, Nurnberg B, Tony To SS. The catalytic phosphoinositol 3-kinase isoform p110delta is required for glioma cell migration and invasion. Eur J Cancer 2012; 48: 149-57.

34 Weigelt B, Warne PH, Lambros MB, Reis-Filho JS, Downward J. PI3K pathway dependencies in endometrioid endometrial cancer cell lines. Clin Cancer Res 2013; 19: 3533-44.

35 Hirsch DS, Shen Y, Dokmanovic M, Wu WJ. pp60c-Src phosphorylates and activates vacuolar protein sorting 34 to mediate cellular transformation. Cancer Res 2010; 70: 5974-83.

36 Ronan B, Flamand O, Vescovi L, Dureuil C, Durand L, Fassy F, et al. A highly potent and selective Vps34 inhibitor alters vesicle trafficking and autophagy. Nat Chem Biol 2014; 10: 1013-9.

37 Liu N, Rowley BR, Bull CO, Schneider C, Haegebarth A, Schatz CA, et al. BAY 80-6946 is a highly selective intravenous PI3K inhibitor with potent $\mathrm{p} 110 \mathrm{alpha}$ and $\mathrm{p} 110$ delta activities in tumor cell lines and xenograft models. Mol Cancer Ther 2013; 12: 2319-30.

38 Barlaam B, Cosulich S, Degorce S, Fitzek M, Green S, Hancox U, et al. Discovery of (R)-8-(1-(3,5-difluorophenylamino)ethyl)-N,N-dimethyl-2morpholino-4-oxo-4H-chrom ene-6-carboxamide (AZD8186): a potent and selective inhibitor of PI3Kbeta and PI3Kdelta for the treatment of PTEN-deficient cancers. J Med Chem 2015; 58: 943-62.

39 Ndubaku CO, Heffron TP, Staben ST, Baumgardner M, Blaquiere N, Bradley E, et al. Discovery of 2-\{3-[2-(1-isopropyl-3-methyl-1H-1,24-triazol-5-yl)-5,6-dihydrobenzo[f]imidazo[1 ,2-d][1,4]oxazepin-9-yl]1H-pyrazol-1-yl\}-2-methylpropanamide (GDC-0032): a beta-sparing phosphoinositide 3-kinase inhibitor with high unbound exposure and robust in vivo antitumor activity. J Med Chem 2013; 56: 4597-610. 